DEPARTAMENTO IJF. ANATOMIA RESCRITIVA DOS ANIMAIS DOMÉSTICOS DIRETOR: Prof. Dr. Orlando M. Paiva

\title{
DISPOSIÇÕES INCOMUNS DA ANSA SPIRALIS DO CÓLON EM BOVINOS AZEBUAdOS *
}

(Unegular patterns of the onso spiralis of the zebu calon)

Orlando M. Paiva

Professor Calediátioo

Vicente Baralli Instrutor

Prosseguindo no estudo do trato digestivo de zebuinos e tendo 1nspecionado, recentemente, os arranjos regulares da onso spiralis (PATVA e BORlLLI, 1963), concentramos, agora, a atenção nas disposições Inus1tadas dêste segmento, que, posto entre a onso proximalis e a onso distalis, forma com elas o colon primum, homólogo do colon oscendens do homem (SUSSDORF, 1901; MARTIN, 1912; ZIETZSCHMANN, 1925; KRItGFR, 1929). A alça espiral, veriflcamos então, baseados cm 1227 observações, descreve um e melo $(9,05 \% \pm 0,82)$, dols $(84,27 \% \pm$ $1,03)$ ou do1s e me1o $(6,68, \% \pm 0,71)$ gyri contriporcles e, en 1gual sequêncla, dois, dols e melo e três gyri contritugales.

Entre as obras gerals, somente as de ELIENBERGER e BAUM (1932) e MARTIN e SCHAUDER (1935) relatam a eventual corrêncla de defeltos no cólon convoluto dos grandes ruminantes.

SMITH e MEADOWS (1956), Invest1gando o comportamento da focal1zada seç̧ão do Intestino, em exemplares de origem europé1a,

* Comunicado a XVII Confertencia Anual da Sociadode Paulista de Madicino Veferinario, realizoda de 8 a 12 de selembro de 1962.

Trabalho ofotuado sob os auspicios do Fundoçbo de Amporo a Posquisa do Estodo de Sto Paulo. 
declaram que nos 735 espécimes vistoriados, 711 ou $96,7, j$ exibiamna ordenada, enbora variasse o número de circuitos, e 24 ou 3,3\% mostravam-na imperfelta. A proporção destas é bastante inferior à indicada por SMITH (1955 a), para carneiros, cêrea de 20, or' dos quals posisuem cólons cujas falhas, afirma SMIIIT (1958), se dividem por três categorias, consoante se situem apenas na última curva de saĺda, em êualquer das voltas à exceção da derradeira, concomitante mente nesta e alguma outra. O próprio SMITH (1955 b) dá relêvo ao fato de que 25, 估 dos aspectos invulgares se localizam no giro cen trffugo externo, apartado dos restantes por larga faixa do mesenté rio comur, contendo linfonodos. Nos bovinos, ao contrárlo, tal es pira, livre de deformidades, não se afasta das ma1s.

Fm nossa anterior e já referida publicação, das 1261 al ças examinadas, 1010 pertencentes a machos e 251 a fêmeas, de várias idades, separamos, conservando-as em formol a 10\%, 34, por patentearem alterações no enrolamento. De todos os casos, 30 do primeiro grupo e 4 do segundo, colhemos desenhos esquemáticos (figs. la31), nêles assinalando as irregularidades e decorrentes des. locamentos, não levados em aprêço para fins de qualificação.

Os vícios de configuração, constantes do material par nós ut1lizado, alcançam $2,77 \% \pm 0,46^{*}$ das viscerais, distribuindo- se, no que toca aos sexos, respectivamente, por $2,97, \pm 0.53$ e $1,60 \% \pm 0,79$ dos indivfduos, diferença de incidência não signifl cante. Alıás, em substitulção ao critério de SMITH, preferimos clas sificá-los, atentos à posição que ocupam, como se inseridos fossem 
Rev. Fac. Med. Vet. S. Paulo - Vol. 7, fasc. 1, 1963-64

em cólons normals. Assim, os desvios das peças com um e melo circuitos proximais e dols distais, inscrevem-se quer sôbre as melas voltas que se avizinham do centro, ou seja, 7 vêzes (Obs. 4,7,23, $27,28,32,33$ ) na segunda, 2 (Obs. 2,20) entre esta e a tercelra, 2. (Obs. 11,26) na primelra e 1 (Obs. 24) na tercelra, quer sô. bre as que dêle se afastam, a saber, 5 vêzes (Obs. 9,12, 13,16,29) na terceira, I vez (Obs. 6) na segunda e 1 (Obs. 17) entre esta e a primetra. Por exceção, 1 vez (Obs. 1) o defelto recal no centro da espiral. As falhas encontradas ell cólons com

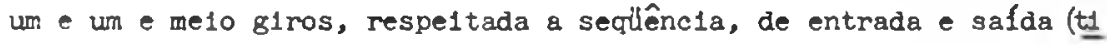
po registrado por SMITH e MEADOWS), surgem 1 vez (Obs. 15) em pleno primelro melo lance concêntrico, 1 (Obs. 19) entre ês. te e o segundo, e 7 vêzes nos melos arcos excêntricos, 1sto $\dot{e}, 4$ (Obs. 8, 18, 30, 34) no segundo, 2 (Obs. 21, 22) no prime1ro, 1 (Obs. 3) em ambos. Enfim, 1 vez (Obs. 14) as modffficações interessaul os dols gêneros de melos lances, colocando-se entre o pl meiro e segundo centrfpetos e no primeiro centrífugo. As incorre ̧̧ões instaladas em alças com duas voltas proxima1s e duas e meia distals aparecer 1 vez (Obs. 5) na tercelra meia espira concên trica, 1 (Obs. 31) entre esta e a quarta, e 1 vez (Obs. 10) no quarto meio c1rcuito excêntrico. Um caso (Obs. 25) foge a con sıderação, por intelramente defe1tuoso.

Multas irregularidades são repetidas (Obs. 8,18/12,13/ 27,28 ) ou pelo menos têm o mesmo caráter (obs. $7,23,27,28,32$, $33 / 9,12,13,16,29 / 8,18,30,34 / 2,4 / 14,19 / 20,24 /$ 21,22); vemo-las, tambén, a atingirem tipos de cólons que lembram os apontados por SMTIH e MEADOWS, como possutindo um e um quarto (Obs. 21, 22, 34) e um e três quartos (Obs. 7, 17) g1ros centrf petos. 
A porcentagem global de peças mal conformadas revela-se, em bovinos azebuados, quase 1dêntica à apurada pelos citados $M$. para os de origem européta, haverdo inclusive apreciável carcela de feitios razoàvelmente coincidentes (Obs. 23,27,28,32/16/30 (34).

Da volta excêntrica termina, ısenta de Imperfelções se. gundo SMITH e MEADOWS, descobrimos livre só a metade final. Pon dere-se, entretanto, que a diversidade de conceltuações relativamen te ao lance en foco invalida o confronto das afirmativas.

$\begin{array}{lllllll}S & \text { U } & \text { M } & \text { M } & \text { A } & \mathbf{R} & \mathrm{Y}\end{array}$

Irregularities in the colling of the anso spiralis of the zebu colon have been observed and classifled. Some deviation was seen in 34 or $2.77 \% \pm 0.45$ out of a total of 1261 speci mens analysed in a previous publication. There was not significant difference betwean sexes.

\section{RFFERÊNCIAS BIBIJOGRÁFICAS}

ELIENBERGLR, W. - BAUA, H. - 1932 - Handbuch der vergleichenden Anatomie der Haustiere. 17 huf. Berlin, Jullus Snringer 
Rer. Fac. Med. Vet. S. Paulo - Vol. 7, fasc. 1, 1963-64

KRUGER, W. - 1929 - D1e vergle1chende Entw1cklungsgeschlchte im Dienste der Lbsung der Homologislerungsproblems an den Darm-und Gekrösabschntten des Menschen und elniger Haussüget1ere (Hhind, Katze, Pferd, Schwein und Wlederkltuer). Z. ges. Anat, $90: 458-548$

MARTIN, P. - 1912 - Lehrbuch der Anatomle der Heartiere. 2. Auf. Bd. 1. Stuttgart, Schickhard \& Ebner

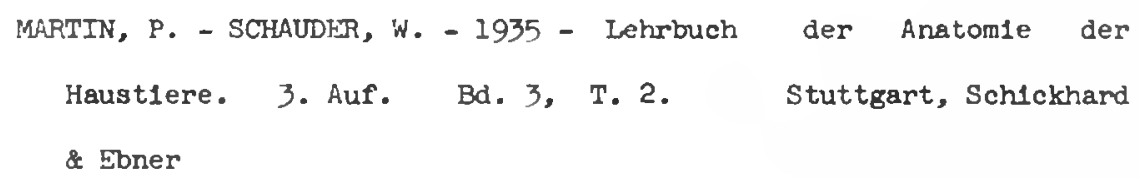


SUSSDORF, M. - 1901 - Homologien der Abschnitte des Dickdarmns und dessen GefHssvertellure. Cit.: EIJENBERGR, W. - BNUM, H. $-1932$

ZIFTZSCHMANN, 0. - 1925 - Der Darmiknal der iüugetiere, eln ver gleichend-anatomisches und entwicklungsieschichtliches Problem. Anat. Anz., S0: 155-172 

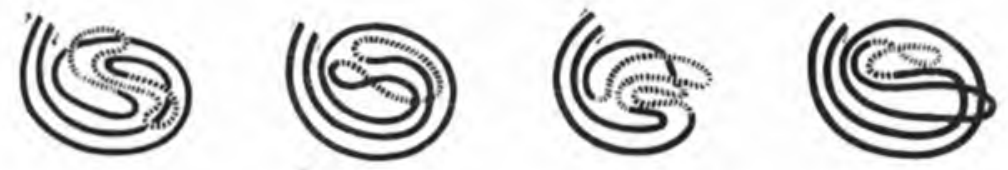

1. Obs. 1

2 - Obs. 2

3. Obs. 3

4. Obs. 4
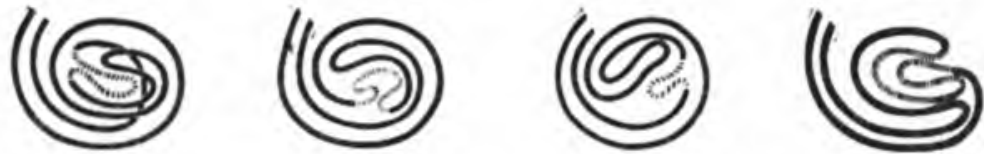

5. Obs. 5

6. Obs. 6

7. Obs. 7

8. Obs. $8 \cdot 18$
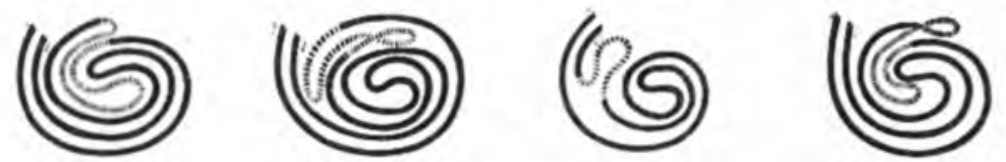

9. Obs. 9

10 . Obs. 10

11 - Obs. 11

12 - Obs. $12 \bullet 13$
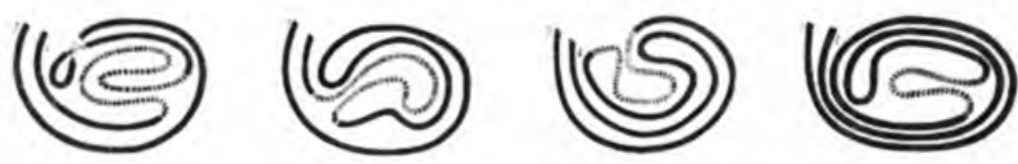

13. Obs. 14

14 - Obs. 15

15. Obs. 16

16. Ob. 17
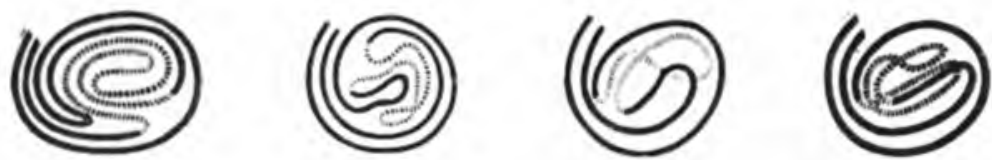

17 . Obs. 19

18 . Obs. 20

19. Obs. 21

m. Obs. 22
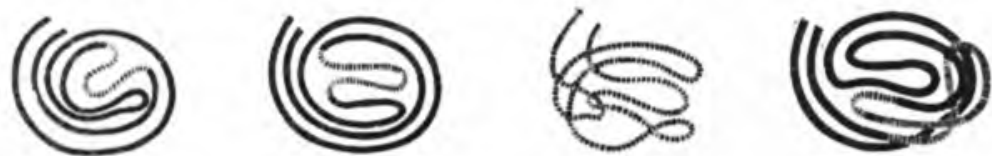

21 - Obs. 23

22 - Obs. 24

23 . Obs. 25

24. Obs. 26
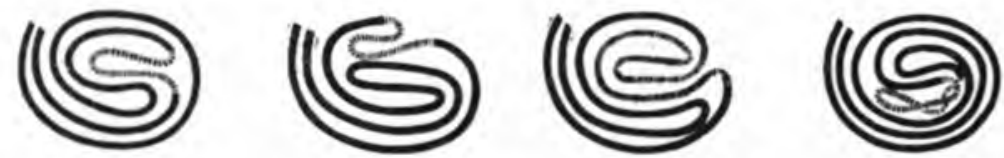

$25 \cdot$ Obs. $27 \cdot 28$

26 . Obs. 29

27 - Obs. 30

20. Obs. 31

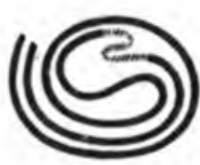

29. Obs. 32

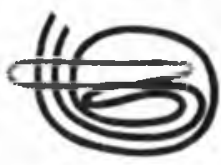

30 . Obs. 33

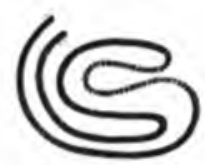

31. Obs. 34

FIGURAS 1 a 31 - Esquemo dos disposicoses incomuns do ansa spiralis do colon de bovinos azebuodos. (Obs. 9, 10, 17 - 34 - (temeas), 